\title{
ON THE AVAILABILITY VERSUS TRANSMITTED POWER OF WIRELESS SENSOR NETWORKS IN RAYLEIGH MULTIPATH FADING ENVIRONMENTS
}

\author{
DEJAN MILIĆ ${ }^{1 \star}$, JELENA ANASTASOV ${ }^{1}$, DANIJEL DOŠIĆ ${ }^{2}$, ALDIS SEFERI $^{2}$ \\ ${ }^{1}$ Faculty of Electronic Engineering, University of Niš, Niš, Serbia \\ ${ }^{2}$ Faculty of Natural Sciences and Mathematics, University of Priština, Kosovska Mitrovica, Serbia
}

\begin{abstract}
This paper presents simulation results for full availability of a wireless sensor network depending on the RF output power of the network nodes in Rayleigh multipath fading environment. Outage probability, relating to the situation in which any one of the sensors is unavailable, is computed by means of numerical simulation, and discussed. The results indicate that the trade-of between the outage probability and the energy efficiency of the network nodes should be sought, resulting in lowest required output power for the highest tolerable outage threshold.
\end{abstract}

Keywords: Wireless sensor networks, Connectivity, Multipath fading, Covering range.

\section{INTRODUCTION}

Wireless sensor networks (WSN), based on collaborative large number of sensor nodes, primarily handle with the information sensing, information processing and then forwarding to the final observer or a base station, (Li et al., 2009). Because of the possibilities that WSNs provide in wireless monitoring and controlling, their use in broadband communications has increased in recent years, (Li et al., 2009; Akyildiz et al., 2002; Patnaik et al., 2015). Today they are widely accepted as vital technology that enhance electrical power systems i.e. the smart grid, etc. (Gungor et al., 2010). Changes in the topology of the sensors' environment lead to changes in sensors' power allocation. In addition, the performance of WSNs is subjected to wireless channel characteristics, namely - multipath fading and shadowing phenomena.

According to gained experimental data, fading environment of WSN can be described as Rayleigh or close to Rayleigh fading. Relying on this, numerous published works have dealt with Rayleigh fading as a main disturbance in WSN leading to signal strength attenuation, (Bergamo \& Mazzini, 2002; Puccinelli \& Haenggi, 2006; Haenggi, 2003; Ren et al., 2011; Kumar \& Lobiyal, 2013; Olofsson et al., 2016). The authors in Bergamo \& Mazzini, (2002) have focused on optimal sensor localization in WSN, taking into account Rayleigh fading influence and possible sensor mobility. They proposed a simple schemes with low energy and computation complexity cost. In Puccinelli \& Haenggi, (2006), the unreliability of sensor network caused by fading phenomena is discussed and adequate measurements and result interpretations are given.

The lifetime of sensors is one of primary concerns in WSN since the sensed data should be delivered to a single sink or specified destination in a certain time limit. According to this, five strategies that balance energy consumption in reaching required sensors' lifetime in fading environment were proposed in Haenggi, (2003).

In addition to fading presence as a dominant nuisance in wireless networks, the shadowing phenomena influence on system performance degradation was considered in Ren et al., (2011); Kumar \& Lobiyal, (2013). As a matter of fact, the impact of Rayleigh fading, path loss and shadowing on error performance was analyzed in Ren et al., (2011). Sensing coverage problem was discussed in Kumar \& Lobiyal, (2013) relating to solutions with Elfes or shadowing sensing model. Furthermore, to define bit error rate as well as packet error rate of specific system, novel adaptive fading statistics in industrial sensor networks were proposed in Olofsson et al., (2016). Simulation model that characterize the wireless channel in industrial environments under Rician fading, was proposed in Gomes et al., (2017).

In this paper, we analyze full availability of a WSNs versus output power of sensors over Rayleigh fading channel. We assume log-distance path loss model in describing signal strength attenuation from node to node communication. Under given circumstances, the outage probability is defined as probability that any one of sensor nodes is unavailable in communication, and appropriate numerical results are given. In order to confirm presented analysis, simulation results are also presented.

The paper is structured as follows. In section 2, specific system model is presented and graphical illustration of coverage area with randomly placed sensors is given. This section also contains description of fading and path-loss model. Simulation setup is explained in section 3, and furthermore numerical and simulation results with appropriate discussion are shown in section 4 . The paper is closed by main concluding remarks given in section 5 . 


\section{SYSTEM MODEL}

The system model assumes that a number of sensors are distributed randomly over a square closed space. Square geometry represents the simplest of cases, but it does not make the generality of the model decrease. In fact, it is relatively simple to modify the simulation model in order to include other geometries. Each sensor node represents a transceiver that incorporates transmitter and receiver blocks. It is assumed that the channels in nodes communication are orthogonal, i.e. that other nodes do not interfere with node-to-node communication. This can be achieved in a number of ways, for example - by using precise time synchronization and scheduling such that each sensor is assigned a time slot in which it transmits its data. Outside of the assigned time slot, the sensor receives the data from other sensors that are within its range and tries to decode and store their messages. If needed, it can resend, or relay, other sensors data so that in perspective, each sensor can communicate with every other sensor, whether directly, or through a number of relays. The sensor nodes acting as relays in this scenario have the role of so-called decode-and-forward (DF) relays, because they fully decode and then repeat the message to other nodes.

In any case, the network can certainly function as completely connected network, or the full availability network. This term denotes a network in which all the nodes can be reached through any one of the nodes, and the data from each sensor can be collected from a single point. In contrast, there might be networks that can not interconnect in such a complete way. For example, one can have two separate distant groups of sensors that are not able to interconnect with one another. In a sense, this can be viewed as a single sensor network with two fully connected clusters. Each cluster exhibits full availability on its own, but is unaware of the existence of the other cluster. Of course, this concept can be extended to multiple clusters in general.

One of the possible realizations of random sensor placement is shown in Fig. 1. This example shows ten sensors placed randomly in the matrix of dimensions $20 \times 20$, representing the square closed-space. The sensor nodes are numbered, so each sensors has its own unique identification number, or address, through which its messages can be identified. It is obvious that the distribution of sensors is not uniform in the space, and there are clusters in which the sensors are closer to each other. Each sensor coverage can be represented by a number of ways, for example - disk model assumes that each sensor can communicate with other sensors that are inside the disk centered at the sensor node, and with fixed radius proportional to sensor output power. However, this model is in many cases too simplistic to accurately describe the performance of sensor networks.

More realistic model takes into account complex propagation effects of the radio waves. Usually, the signal rarely propagates directly towards the destination node. In practice, emitted radio waves spread into all directions and reflect of objects in the closed space. Therefore, at the destination, we have a number

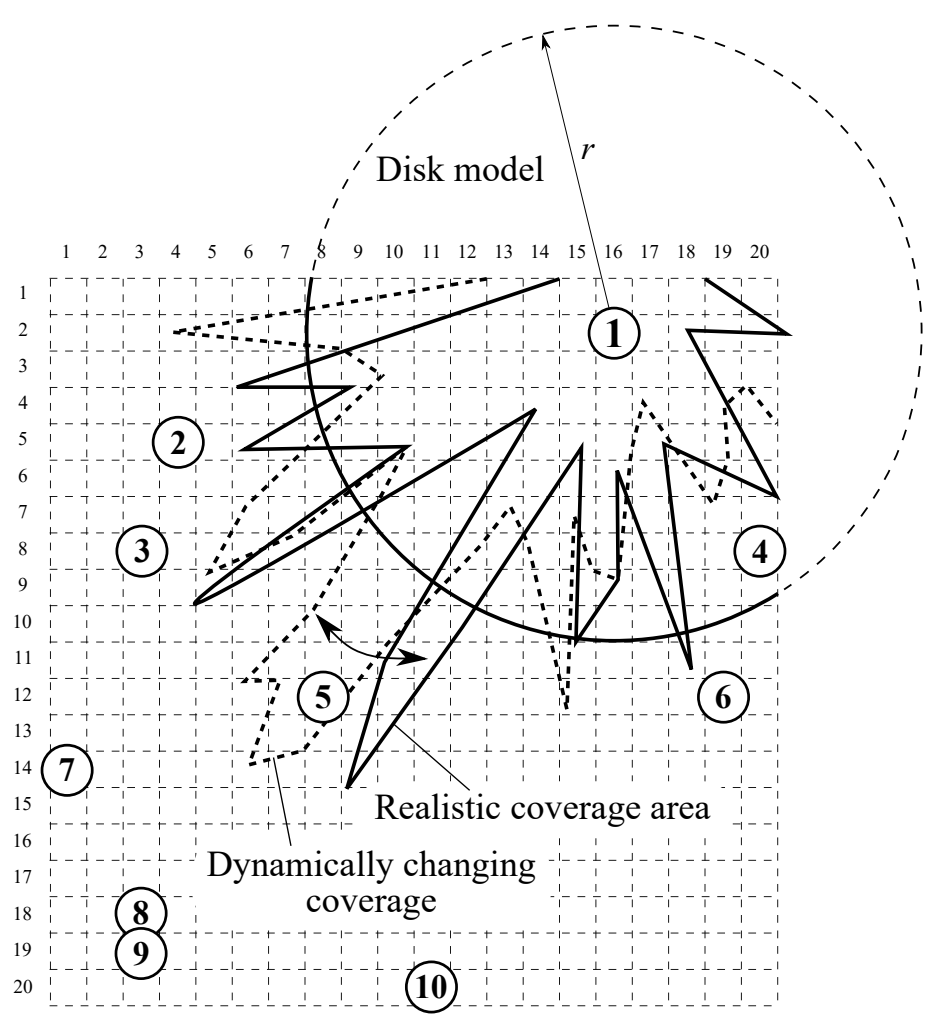

Figure 1. A realization of 10 random sensors distribution in $20 \times 20$ matrix, and illustration of coverage models.

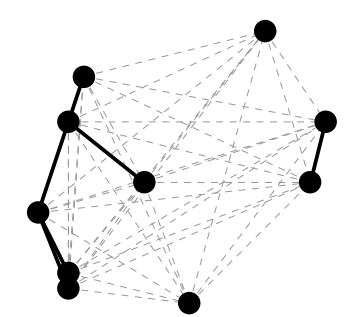

(a) $r=7$

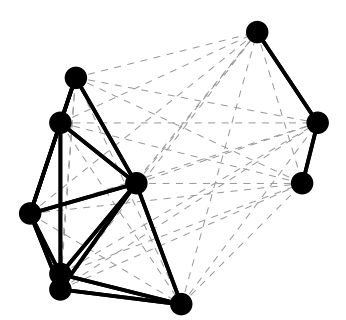

(b) $r=10$

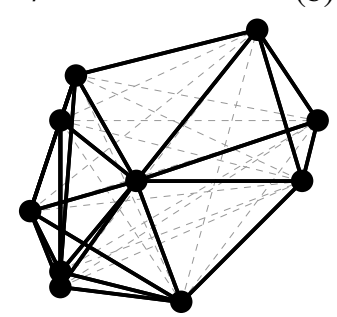

(c) $r=13$

Figure 2. Illustration of sensors connectivity using simple disk coverage model with indicated diameter $r$

of radio waves coming from different directions, reflected from a number of objects, and converging onto the receiver antenna. Each of the waves have different phase, according to its path, and all the radio waves with different phases and amplitudes interfere with one another, producing the signal level at the receiver antenna. Although it is possible to accurately simulate the realistic effects of such propagation, it is a very complex task. Instead, the propagation effects are modeled on the basis of statistical description, which is in turn based on the series of precise signal level mea- 
surements. Realistic node coverage area has more or less irregular shape, as illustrated in Fig. 1. Also, as conditions change over time, due to differences in temperatures, presence or absence of people, or slight changes in geometry and positions of surrounding objects, coverage area changes its shape dynamically. In such conditions, stochastic fading models are well-suited for performance analysis of wireless networks.

One of the most commonly used multipath fading models is the Rayleigh model. It assumes that the received signal envelope $R$ over the wireless channel has Rayleigh probability density function (PDF), (Panić et al., 2013):

$$
p_{R}(r)=\frac{2 r}{\Omega} e^{-r^{2} / \Omega}
$$

where $\Omega$ is the mean-square value of the envelope. The Rayleigh model has been used extensively in research literature to model the fading channels in wireless communications, including the sensor networks. It is considered the simplest of fading channel models, yet its potentials are not yet exhausted as the sensor networks represent complex systems, and the required computational complexity for the performance analysis is not neglectable.

Each sensor tries to demodulate the received signal according to the modulation format used. To facilitate demodulation, each transmitted packet of data has a pre-determined structure. Usually, it starts with a pre-amble, which is a fixed length sequence of alternating bits. Receiver uses the pre-amble to set up the time synchronization circuits and estimate the signal level, and therefore estimate the signal-to-noise ratio (SNR). Most of the noise in the channel originates from the thermal noise in the pre-amplifier circuitry of the receiver. Therefore, at the constant temperature, the noise power spectrum density is also constant. Accordingly, a lot of wireless integrated circuits include a digital thermometer, and the receiver is able to estimate the noise levels based on the temperature and receiver bandwidth. During the pre-amble period, receiver estimates the Received Signal Strength Indicator (RSSI) and SNR values. If the SNR is above the pre-determined theoretical threshold value, the receiver concludes that it is able to successfully decode the message, otherwise the rest of the message is ignored.

Following the pre-amble, there is a synchro-sequence, used to delimit the pre-amble from the useful data in a synchronous way. Address sequence identifying the transmitting sensor node follows next, accompanied by the data message. Finally, the message ends with cyclic-redundancy check (CRC) byte enabling transfer error detection. The message can be forward-error correction (FEC) coded, allowing error correction in addition to detection of errors, but this is usually optional.

After this review of the packet format, we continue with the appropriate simple model of receiver: if the SNR is above the threshold, the receiver is able to decode the message with very high probability of success. Otherwise, successful decoding can not be expected and the receiving node does not attempt to do so.
Packer-error rate is then:

$$
\text { PER }= \begin{cases}0, & \text { SNR }>t_{h} \\ 1, & \text { SNR } \leq t_{h}\end{cases}
$$

Therefore, the threshold is very significant parameter for the sensors, and it defines the sensitivity of the nodes. If we assume that all the nodes transmit at the same pre-determined signal level, the node coverage is determined by the sensitivity threshold. If the SNR at the receiver is lower than the threshold, we say that the receiver experiences signal outage.

It is of vital interest to maintain the signal levels above the outage threshold. However, this can always be guaranteed if the nodes transmit at excessively high power. Of course this is not practical, and the nodes usually transmit at levels just above the required level, including a safety margin. Subsequently, outagefree operation can not be guaranteed, because the signals exhibit dynamic behavior, as discussed earlier. Instead of strict guarantee, probabilistic measures are used and the probability of outage is kept under the pre-defined low percentage value.

Another important parameter in considering the node coverage is the path loss. Obviously, the signal strength decreases with increasing distance from the transmitter, and this decrease - or loss, should also be included into the model, regardless that the distances are relatively small. There are multiple propagation models that take path loss into account differently, but ultimately the models are based on experience and analysis of large measurement datasets. For the sake of simplicity of formulation, we use the log-distance path loss model for indoor environments. When considering relevant parameters for the specific system model, we get the following form the path loss $P_{L}$ at distance $d$ from the transmitter (Ren et al., 2011):

$$
P_{L}[\mathrm{~dB}]=P_{L_{0}}+10 \gamma \log _{10} \frac{d}{d_{0}},
$$

where the distance $d_{0}$ is the reference distance (usually $1 \mathrm{~m}$ ), $P_{L_{0}}$ is the loss over reference distance, and $\gamma$ represents the path loss exponent. The exponent $\gamma$ can have different values, depending on the characteristics of the propagation environment, but is usually in the range of $2.0-3.0$ for the closed-space office environments. We adopt the value of 2.5 for further simulations. Therefore, signal power $P_{\mathrm{rx}}$ at the receiver is:

$$
P_{\mathrm{rx}}=P_{0}\left(\frac{d_{0}}{d}\right)^{\gamma}
$$

where the power $P_{0}$ at close distance $d_{0}$ is a constant fraction of the total output power $P_{0}=\alpha P_{\mathrm{tx}}$.

\section{SIMULATION SETUP}

Based on the model from the previous section, we set up a simulation to numerically evaluate the availability of the network. In first step of the simulation, it is necessary to calculate mutual distances between the sensors. As the number of sensors 
is $N=10$, it is obvious that there are 45 interconnections in total, or $N(N-1) / 2$. For the specific case we use the node distribution shown in Fig. 1. Euclidean distances between nodes:

$$
d_{i, j}=\left(\left(x_{i}-x_{j}\right)^{2}+\left(y_{i}-y_{j}\right)^{2}\right)^{1 / 2},
$$

are calculated, arranged in increasing order, and shown in Fig. 3.

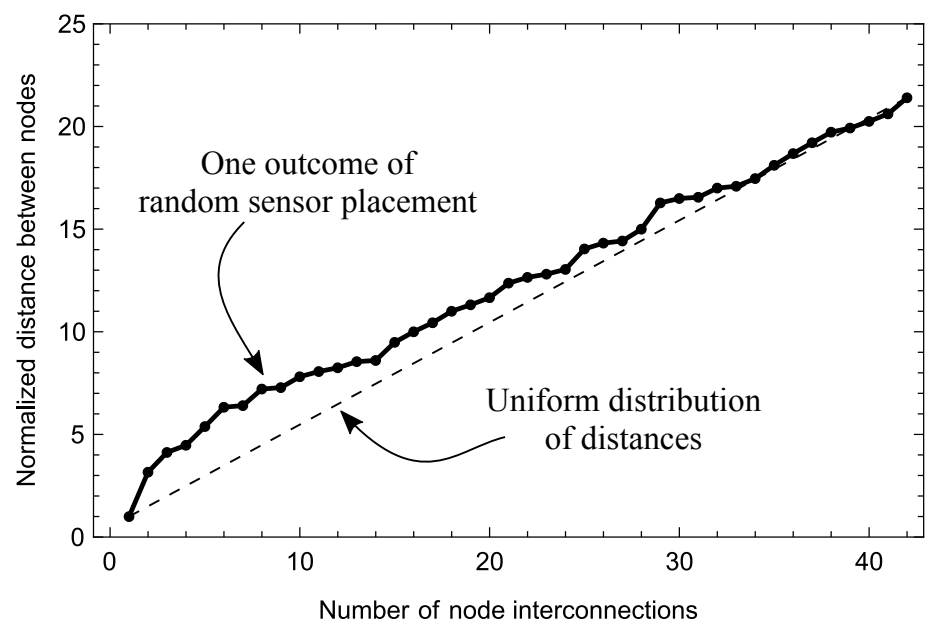

Figure 3. Distribution of internode distances for the sensor distribution in Fig. 1.

We furhter assume that all nodes transmitt at the same output power. According to path-loss model, mean signal power that from one node reaches the other node is given by (4). Due to multipath fading, signal amplitude can vary according to (1). Since signal power relates to amplitude $R$ as: $P_{\mathrm{rx}}=R^{2} / 2$, we need to use $\Omega=P_{\mathrm{rx}} / 2$. Single outcome of multipath fading affects specific link, or interconnection between two nodes. According to (2), if the resulting signal power at the receiver is above the threshold, we consider the link to be functional, otherwise the particular link is down.

When the procedure of simulating the individual links is repeated for each of the 45 interconnections, the links are divided into groups of passed and failed, and the clusters of interconnected nodes are identified. If the total number of clusters in the network is exactly one, than the network is fully available. In other cases, we have more than one separate clusters that can not communicate with each other. Therefore, the number of clusters is also a random variable and can have different values for the specific fading realizations. With sensor placement as in Fig. 1, and for a number of 1000 simulation runs, we have computed the number of clusters for different outage threshold values, and the results are shown in Fig. 4. Results indicate that the low threshold values would result mostly in a single cluster, while higher thresholds favor multiple clusters. Therefore, it is obvious that the receiver sensitivity should be as high as possible, allowing lower outage thresholds and thus higher availability of nodes. In this point of view, threshold corresponds reciprocally to receiver sensitivity. The higher the sensitivity of the receiver is, the lower is the outage threshold value.

The results can also be interpreted based on the output power of nodes. If we assume that the receiver sensitivity is fixed, and is

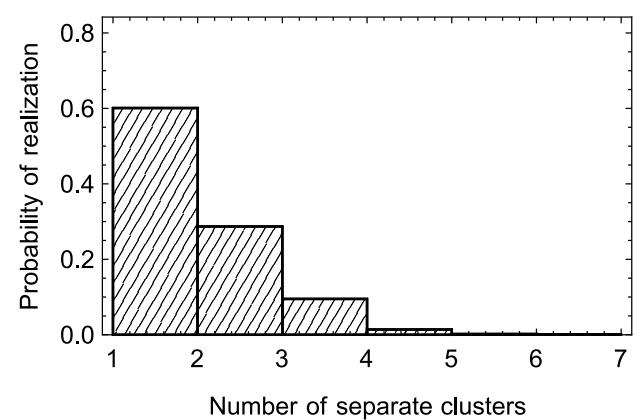

(a) $t_{h}=-25 \mathrm{~dB}$

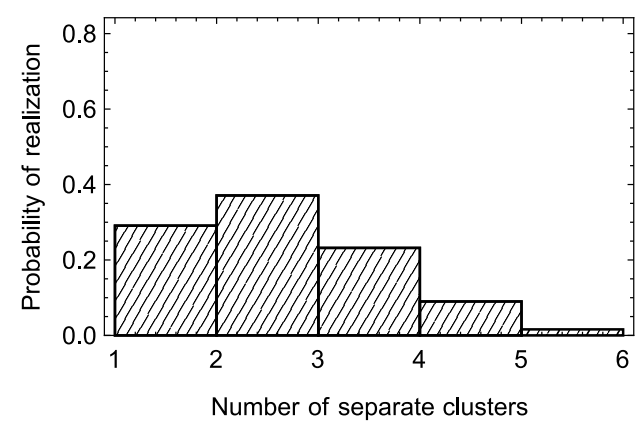

(b) $t_{h}=-24 \mathrm{~dB}$

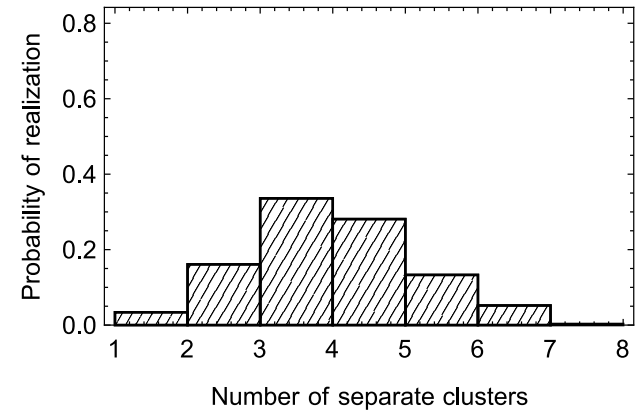

(c) $t_{h}=-22 \mathrm{~dB}$

Figure 4. Example of cluster distribution for different outage thresholds

not subject to changes, than the output power should be increased to allow higher signal levels at reception. From this point of view, the outage threshold is fixed, and the values of $t_{h}$ indicate the relative position of threshold in comparison to node output power. In other words, absolute values of $t_{h}$ indicate the output power level over the outage threshold value.

\section{NUMERICAL RESULTS AND DISCUSSION}

Simulations run until the total number of iterations is reached, or the number of positive outcomes is higher than 20. We take the fully available network as a positive outcome. In this way, the unnecessary usage of computer resources is avoided for the cases where percentage of positive outcomes is relatively high, i.e. there is no need to continue simulations when the result already reaches certain degree of precision. On the other hand, for the rare occurrences of positive outcomes, the maximum number of simulations will be run without reaching the requested precision. 


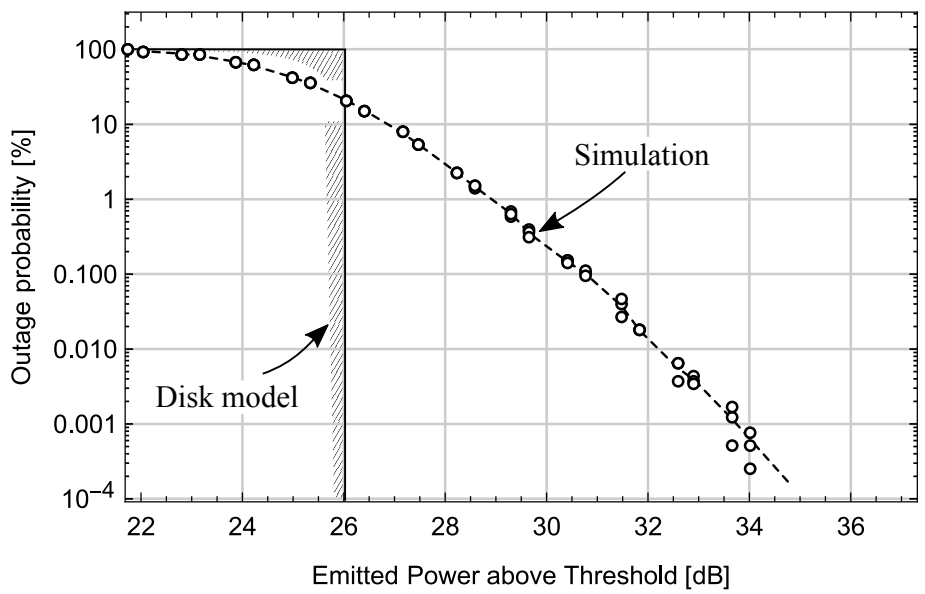

Figure 5. Outage probability percentage versus node output power

We denote the ratio of emitted power to the outage threshold as:

$$
\xi=\frac{P_{0}}{t_{h}}
$$

Power threshold at the receiver is taken to be the limit of the node sensitivity $t_{h}=P_{\mathrm{x}}$, but can also be set above that value as a safety margin. Therefore, $\xi$ represents the portion of emitted power level that is above the receiver sensitivity limit. According to (4) and the disk model, we get:

$$
\xi[\mathrm{dB}]=10 \gamma \log _{10} \frac{\mathrm{d}_{(2 \rightarrow 1)}}{\mathrm{d}_{0}},
$$

From the Figs (1) and (2), we conclude that the crossing point between the two clusters and fully available network occurs when the disk radius reaches $d_{(2 \rightarrow 1)}=11$, connecting the node 6 to nodes 5 and 10. Therefore, (7) predicts that the emitted power should be at least $26.03 \mathrm{~dB}$ above the node sensitivity in order to form the network with full availability. Furthermore, according to the model there is a sharp transition step between the two clusters and fully available network, and the network is perfectly functional after crossing this transition.

However, propagation model that takes into account the Rayleigh fading predicts more detailed results than the deterministic disk model. Main difference in predictions is that there is not an abrupt transition between the multiple clusters and full availability. Instead, the stochastic model exhibits smooth transition. The model uses continuous outage probability as a measure of network availability, which is the probability that the network will not be fully available. Thus, the network is better connected when the outage probability is lower.

When the transmitted power is $26 \mathrm{~dB}$ over the node sensitivity, the outage probability reads at about $20 \%$, meaning that the network may not be available for $20 \%$ of the time. If we need this outage probability to be much lower, for example: $1 \%$, we need higher output power of $29 \mathrm{~dB}$, which is exactly double the required value we got for the disk model. From the data we estimate that after the break point, increase in power by factor 1.65 , i.e. power increase by $65 \%$ leads to ten-fold reduction in outage probability.
For the break point we take the output power for which the outage probability is $50 \%$, and for the specific case this value is $24.6 \mathrm{~dB}$.

For other particular distributions of sensor nodes, one should expect slightly different break points, and also somewhat different slopes of the outage probability curves, although the general shape of the curves will be similar. This is due to the fact that the maximal cluster distance is limited by the closed-space geometry and the density of sensor nodes. Disk model can serve as a rough estimate of the break point, providing that a critical link connecting two clusters into a single cluster network can be identified.

\section{CONCLUSION}

We have set up a simulation model that is capable of describing the connectivity of wireless sensor networks in indoor spaces with multipath Rayleigh fading. Simulation uses log-distance model to account for average signal decay with distance from network node. Although simple in its assumptions, simulation model is capable of giving insight into complex interplay between the network nodes in a dynamical environment. The numerical results obtained are focused on the full availability of the network, and the transmitter power that is required to attain it. Results of simulation are encouraging, and more efforts should be invested into deriving the analytical model to match the results. Outage probability versus the transmitter power curve can be obtained by means of simulation for any particular distribution of sensors. The results indicate that the trade-of between the outage probability and the energy efficiency of the network nodes should be sought, resulting in lowest required output power for the highest tolerable outage threshold.

\section{ACKNOWLEDGEMENT}

D. Milić and J. Anastasov were supported in part by the Ministry of Science of Republic of Serbia under grant III44006.

\section{REFERENCES}

Akyildiz, I. F., Su, W., Sankarasubramaniam, Y., \& Cayirci, E. 2002. Wireless sensor networks: A survey. Computer networks, 38(4), pp. 393-422.

Bergamo, P. \& Mazzini, G. 2002. Localization in sensor networks with fading and mobility. In: The 13th IEEE International Symposium on Personal, Indoor and Mobile Radio Communications, IEEE, pp. 750-754 vol. 2.

Gomes, R. D., Queiroz, D. V., Fonseca, I. E., \& Alencar, M. S. 2017. A simulation model for industrial multi-channel wireless sensor networks. Journal of Communication and Information Systems, 32(1).

Gungor, V. C., Lu, B., \& Hancke, G. P. 2010. Opportunities and challenges of wireless sensor networks in smart grid. IEEE transactions on industrial electronics, 57(10), pp. 3557-3564.

Haenggi, M. 2003. Energy-balancing strategies for wireless sensor networks. In: Proceedings of the International Symposium Circuits and Systems ISCAS'03, IEEE, pp. 4-4 vol. 4. 
Kumar, S. \& Lobiyal, D. 2013. Sensing coverage prediction for wireless sensor networks in shadowed and multipath environment. The Scientific World Journal, 2013.

Li, J., Andrew, L. L., Foh, C. H., Zukerman, M., \& Chen, H.-H. 2009. Connectivity, coverage and placement in wireless sensor networks. Sensors, 9(10), pp. 7664-7693. Pmid:22408474

Olofsson, T., Ahlén, A., \& Gidlund, M. 2016. Modeling of the fading statistics of wireless sensor network channels in industrial environments. IEEE Transactions on Signal Processing, 64(12), pp. 3021-3034.

Panić, S., Stefanović, M., Anastasov, J., \& Spalević, P. 2013. Fading and interference mitigation in wireless communications.
CRC Press.

Patnaik, S., Li, X., \& Yang, Y.-M. 2015. Recent Development in Wireless Sensor and Ad-hoc Networks. Springer.

Puccinelli, D. \& Haenggi, M. 2006. Multipath fading in wireless sensor networks: Measurements and interpretation. In: Proceedings of the 2006 international conference on Wireless communications and mobile computing, ACM, pp. 1039-1044 .

Ren, Z., Wang, G., Chen, Q., \& Li, H. 2011. Modelling and simulation of Rayleigh fading, path loss, and shadowing fading for wireless mobile networks. Simulation Modelling Practice and Theory, 19(2), pp. 626-637. 